\title{
Incidence and risk of anemia in type-2 diabetic patients in the absence of renal impairment
}

\author{
Babatunde Ishola Adejumo ${ }^{1}$, Uchechukwu Dimkpa ${ }^{2^{*}}$, Chinwe Obianuju Ewenighi $^{3}$, \\ Abduffatah Adekunle Onifade ${ }^{4}$, Azukaego Thomas Mokogwu ${ }^{5}$, Tosan Amos Erhabor ${ }^{6}$, \\ Esmond Ukatu ${ }^{7}$, Isaac Nwoye Nnatuanya ${ }^{8}$ \\ ${ }^{1}$ Medical Laboratory Sciences Department, University of Benin, Benin City, Nigeria \\ ${ }^{2}$ Physiology Department, Nnewi Campus, Nnamdi Azikiwe University, Awka, Nigeria; \\ *Corresponding Author: positivedoings@yahoo.com \\ ${ }^{3}$ Medical Laboratory Sciences Department, Ebonyi State University, Abakaliki, Nigeria \\ ${ }^{4}$ Department of Chemical Pathology, College of Medicine, University of Ibadan, Ibadan, Nigeria \\ ${ }^{5}$ Department of Chemical Pathology, School of Clinical Medicine, Igbinedion University, Okada, Nigeria \\ ${ }^{6}$ Research and Training Department, Medical Laboratory Science Council of Nigeria, Abuja, Nigeria \\ ${ }^{7}$ Education and Research Department, May and Baker Plc, Lagos, Nigeria \\ ${ }^{8}$ Department of Chemical Pathology/Toxicology, Faculty of Medical Lab Sciences, Elele Campus, Madonna University, Okija, Nigeria
}

Received 15 February 2012; revised 20 March 2012; accepted 10 April 2012

\section{ABSTRACT}

Aim: To determine the incidence of anemia in type-2 diabetic patients with normal renal function and assess the association of other factors related to diabetes with the risk of anemia. Methods: The study group comprised 72 patients with type-2 diabetes. Patients were divided into groups according to glycemic control, gender and age. Serum creatinine, hematocrit, hemoglobin concentration, blood glucose and glycated hemoglobin (HbA1c) of subjects were measured. The presence of anemia was defined by a hemoglobin level $<13.0 \mathrm{~g} / \mathrm{dL}$ in men and $<12.0 \mathrm{~g} / \mathrm{dL}$ in women. Normal renal function (i.e. absence of renal impairment) was defined as serum creatinine level $<1.5 \mathrm{mg} / \mathrm{dL}$. Results: $15.3 \%$ of the patients had anemia. The odds of developing anemia was higher in patients with poorly controlled diabetes (HbA1c > 7.5\%) compared to those with controlled diabetes $(\leq 7.5 \%)$ and in patients of age $\geq 60 \mathrm{yrs}$ compared to those of age $<60 \mathrm{yrs}(p<0.05)$. The odds of anemia was similar in males and females $(p=0.26)$. Conclusion: This study indicated that poor glycemic control and old age are associated with the incidence of anemia in type-2 diabetics with normal renal function. Our findings suggest that treatment criteria for diabetes should include routine hematological tests and take into consideration the inevitable consequences of ageing, and poor glycemic control.

Keywords: Anemia; Type-2 Diabetes Mellitus;
Glycemic Control; Ageing; Gender

\section{INTRODUCTION}

Anemia is the most common blood disorder and a common finding in patients with diabetes [1]. It is also considered as a key indicator of chronic kidney disease and an important cardiovascular risk factor [2,3]. Previous studies have shown that the incidence of anemia in diabetic patients is mostly associated with the presence of renal insufficiency. Thus, patients with diabetes have a greater degree of anemia for their level of renal impairment than non-diabetic patients presenting with other causes of renal failure [1-5]. Factors suggested as the reason for the earlier onset of anemia in patients with diabetes, include severe symptomatic autonomic neuropathy, causing efferent sympathetic denervation of the kidney and loss of appropriate erythropoietin; damage to the renal interstitium; systemic inflammation; and inhibition of erythropoietin release [6].

In spite of the plethora of reports on the presence of anemia in diabetic patients with renal insufficiency, limited study [7] exists on the incidence of anemia in diabetics prior to the evidence of renal impairment. This may explain why most diabetic patients with normal renal function are rarely tested for anemia. The need for more studies on incidence of anemia in diabetic patients prior to renal impairment has therefore become imperative, in order to increase the level of awareness and understanding of anemia amongst diabetic patients. In addition, it is not certain whether hemorheological effects of other risk factors related to diabetes mellitus may contribute to the greater incidence of anemia usually ob- 
served in diabetic patients irrespective of renal condition. We believe such knowledge may help patients ascertain the impact of anemia on their quality of life, and lead to opportunities for developing interventions to optimize outcomes in diabetic patients.

In the present study therefore, we aimed at demonstrating the incidence and risk of anemia in type- 2 diabetic patients with normal renal function. In addition, the incidence and risk of anemia in these patients were assessed according to gender, age, and glycemic control statuses.

\section{METHODS}

\subsection{Subjects}

This cross sectional study comprised 72 type- 2 diabetic patients with normal renal functions recruited from diabetes clinics of the University of Benin Teaching Hospital, Benin city and Igbinedion University Teaching Hospital, Okada, all in Edo state, Nigeria. Diabetes was diagnosed by participant's self-report, and glycated hemoglobin values $>6.5 \%$. The diabetic patients were divided into groups according to: 1) glycemic control [patients with controlled diabetes $(\mathrm{n}=32)$ and those with poorly controlled diabetes $(\mathrm{n}=40)]$; 2 ) gender (males, $\mathrm{n}=34$;

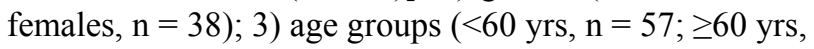
$\mathrm{n}=15$ ). The controlled diabetic group comprised those whose glycated hemoglobin concentration (HbAlc) was $\leq 7.5 \%$. The poorly controlled diabetic comprised those whose HbA1c level was $>7.5 \%$. Group designation, patients' demographics, and clinical features are listed in Table 1.

To be included in the study all patients had to be 18 years of age or older and without renal insufficiency (serum creatinine level of $<1.5 \mathrm{mg} / \mathrm{dL}$ ). Exclusion criteria included those who had history of unstable cardiovascular and peripheral diseases; those with chronic illnesses; those with recent blood loss or donated blood recently; those who have hemolytic anemia or genetic differences in the hemoglobin molecule (hemoglobinopathy) such as sickle-cell disease and other systemic disorders that could result in anemia. Blood were collected from the subjects after obtaining permission from the hospital authority, and under aseptic conditions using fresh needles and syringes and sterile containers. Blood samples were collected into floride oxalate and EDTA bottles for serum creatinine, hematocrit, hemoglobin concentration, fasting blood glucose (FBG), and glycated hemoglobin measurements respectively. The presence of anemia was defined by a hemoglobin level $<13.0 \mathrm{~g} / \mathrm{dL}$ in men and $<12.0 \mathrm{~g} / \mathrm{dL}$ in women based on definition of World Health Organization (WHO) [8]. Fasting blood glucose was estimated using the glucose oxidase method (Randox, United Kingdom). Glycated hemoglobin A1C was assessed by ion exchange chromatography method (DIALAB, Gieselhaft, Germany). The Research and Ethics Committees of the two institutions approved the study and all the subjects gave their written informed consent prior to the study.

\subsection{Statistical Analysis}

Descriptive data are presented as means and standard deviations (SD). Data analysis between two groups was compared using two-tailed independent sample t-test. Two-tailed Pearson's partial correlation coefficient was used to determine age-adjusted correlations between variables. Logistic regression analysis was used for the analysis of associations between anemia and independent variables. All statistics were done using SPSS for Windows (Version 16.0). Significance was set at $\mathrm{p}<0.05$.

\section{RESULTS}

Demographic and clinical characteristics of patients are as shown in Table 1. Mean hemoglobin values were significantly higher in persons with controlled diabetes compared to persons with poorly controlled diabetes $(\mathrm{p}<$ 0.001 ) and in patients of age $<60$ yrs compared to those who are $\geq 60$ yrs $(p<0.05)$. Significantly higher hematocrit was noted in diabetic males and in patients

Table 1. Demographic and clinical characteristics of subjects.

\begin{tabular}{lcccc}
\hline & $\begin{array}{c}\text { All Diabetics } \\
(\mathrm{n}=144)\end{array}$ & $\begin{array}{c}\text { Poorly Controlled Diabetes }(\mathrm{n}=80) \\
\text { vs. Controlled Diabetes }(\mathrm{n}=64)\end{array}$ & $\begin{array}{c}\text { Diabetic Males }(\mathrm{n}=68) \\
\text { vs. Diabetic Females }(\mathrm{n}=76)\end{array}$ & $\begin{array}{c}\text { Diabetics } \geq 60 \text { yrs }(\mathrm{n}=30) \\
\text { vs. Diabetics }<60 \text { yrs }(\mathrm{n}=114)\end{array}$ \\
\hline Age (yrs) & $48.8 \pm 9.09$ & $48.5 \pm 9.02$ vs. $49.2 \pm 9.22$ & $49.2 \pm 10.32$ vs. $48.3 \pm 7.86$ & $59.4 \pm 5.55$ vs. $45.6 \pm 7.44^{* * *}$ \\
HbA1c (\%) & $11.7 \pm 5.77$ & $15.1 \pm 5.85^{* * *}$ vs. $7.5 \pm 0.52$ & $10.2 \pm 4.65$ vs. $13.0 \pm 6.35^{*}$ & $13.4 \pm 7.01$ vs. $11.3 \pm 5.40$ \\
FBG (mg/dl) & $167.6 \pm 48.18$ & $185.7 \pm 53.02^{* * *}$ vs. $145.0 \pm 28.49$ & $160.8 \pm 37.27$ vs. $173.7 \pm 55.72$ & $175.5 \pm 65.56$ vs. $165.5 \pm 43.23$ \\
SCr (mg/dl) & $0.95 \pm 0.29$ & $1.02 \pm 0.27^{*}$ vs. $0.87 \pm 0.28$ & $0.93 \pm 0.28$ vs. $0.97 \pm 0.29$ & $1.0 \pm 0.24$ vs. $0.9 \pm 0.30$ \\
Hemoglobin (g/dl) & $13.1 \pm 1.02$ & $12.7 \pm 0.75$ vs. $13.7 \pm 1.09^{* * *}$ & $13.4 \pm 0.91$ vs. $12.9 \pm 1.07$ & $12.4 \pm 0.82$ vs. $13.2 \pm 1.15^{*}$ \\
Hematocrit (\%) & $39.6 \pm 3.09$ & $38.6 \pm 2.88$ vs. $40.9 \pm 2.82^{* * *}$ & $40.4 \pm 3.01^{*}$ vs. $38.9 \pm 2.98$ & $39.0 \pm 4.21$ vs. $39.4 \pm 3.22$ \\
\hline
\end{tabular}

Abbreviations: $\mathrm{HbA} 1 \mathrm{c}=$ Glycated hemoglobin concentration; FGB $=$ fasting blood glucose; $\mathrm{SCr}=\mathrm{Serum}$ creatinine $;{ }^{*} \mathrm{p}<0.05 ;{ }^{* *} \mathrm{p}<0.01 ;{ }^{* * *} \mathrm{p}<0.001$. Data are mean \pm standard deviation. 
with controlled diabetes compared to females and those with poorly controlled diabetes $(\mathrm{p}<0.001$ and $\mathrm{p}<0.05)$ respectively. On the other hand, mean hemoglobin indicated no gender differences and hematocrit indicated no age differences. Eleven of the 72 diabetic patients presented with anemia representing $15.3 \%$ incidence of anemia (Figure 1). 25\% $(\mathrm{n}=10)$ of the patients with poorly controlled diabetes; $3.1 \%(n=1)$ of those with controlled diabetes; $20.6 \%(\mathrm{n}=7)$ of the diabetic men; $10.5 \%(n=4)$ of the diabetic women; $35.7 \%(n=5)$ of the older patients of age $\geq 60 \mathrm{yrs}$ and $10.3 \%(\mathrm{n}=6)$ of patients of age $<60 \mathrm{yrs}$, were anemic.

Age and sex adjusted Pearson's correlation analysis indicated strong and significant negative correlations between glycated $\mathrm{HbAlc}$ and the indicator variables of anemia: hemoglobin concentration $(r=-0.34, p<0.01)$ and hematocrit $(r=-0.30, p=0.01)$. Similar trend was observed between fasting blood glucose and hemoglobin concentration $(\mathrm{r}=-0.30, \mathrm{p}=0.01)$ and hematocrit $(\mathrm{r}=$ $-0.31, \mathrm{p}<0.01)$. We performed logistic regression analysis to evaluate the odds of developing anemia according to glycemic control, gender, and age statuses of patients. The age-adjusted logistic regression analysis (Table 2) revealed that patients who had poorly controlled diabetes were at higher risk of anemia than those with controlled diabetes $(\mathrm{p}<0.05)$; and that the odds of anemia was similar between males and females $(\mathrm{p}=0.26)$. A sex-adjusted logistic regression also indicated that patients of age $\geq 60 \mathrm{yrs}$ were at greater odds of developing anemia compared to those of $<60 \mathrm{yrs}$ of age $(\mathrm{p}<0.05)$.

\section{DISCUSSION}

In the present cross-sectional study, patients had high incidence of anemia (15.3\%). Interestingly, the parameters for diagnosing diabetes (HbAlc and FBG) had significant, negative correlations with the indicator variables of anemia. Scanty information exists on the incidence of anemia amongst diabetics without renal insufficiency in Nigeria. A previous study by Bonakdara et al. [9] reported that $7.2 \%$ of diabetics with normal renal function had anemia. The higher incidence of anemia in our study may be due to small number of studied subjects, which was largely those who had poorly controlled diabetes, who may be susceptible to impaired erythropoietin production and release due to diabetic neuropathy [7,10]. In contrast, prevalences of $20 \%$ and $19.6 \%$ have been reported respectively in diabetics with renal insufficiency by other studies $[1,9]$.

It is not known whether the incidence of anemia in diabetic patients is dependent on hemorheological effects of other risk factors related to diabetes mellitus. We therefore evaluated the risk of anemia in the diabetic

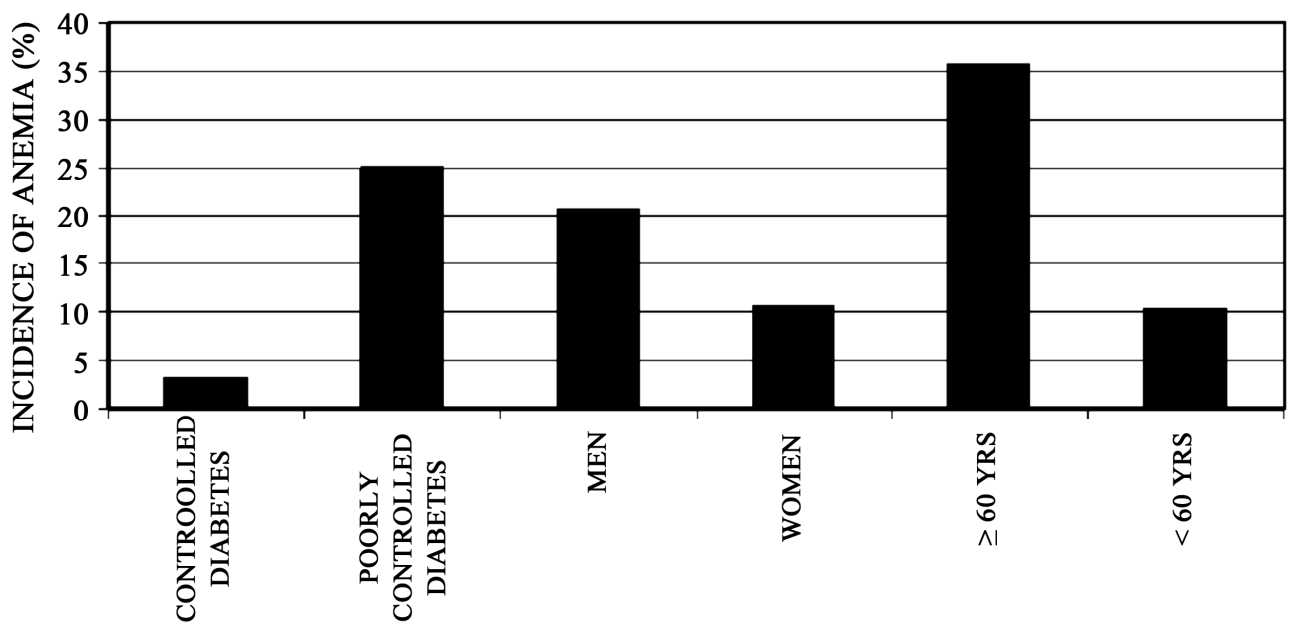

Figure 1. The incidence of anemia in diabetic patients according to glycemic control, gender and age categories.

Table 2. Age-adjusted logistic regression between incidence of anemia and selected risk factors.

\begin{tabular}{ccc}
\hline & Unadjusted OR $(95 \% \mathrm{CI})$ & Adjusted OR $(95 \% \mathrm{CI})$ \\
\hline Glycemic Control Status & $10.3(1.24-85.75) ; \mathrm{p}<0.05$ & $10.8(1.28-91.20) ; \mathrm{p}<0.05^{*}$ \\
Gender & $2.20(0.58-8.31) ; \mathrm{p}=0.24$ & $1.04(0.97-1.12) ; \mathrm{p}=0.26^{*}$ \\
Age Category & $4.25(1.08-16.67) ; \mathrm{p}<0.05$ & $4.13(1.03-16.49) ; \mathrm{p}<0.05^{\mathrm{a}}$ \\
\hline
\end{tabular}

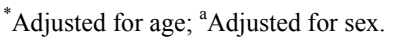


cohorts with regard to age, sex and glycemic control of the patients. Our findings indicated that patients who have poorly controlled diabetes were at greater risk of anemia than those with controlled diabetes. A previous study on diabetic patients with normal renal functions has shown that longstanding poorly controlled diabetes is associated with normocytic normochromic anemia [7]. The mechanisms behind the greater risk of anemia in poorly controlled diabetes than in controlled diabetes are not very clear. However, it has been established that diabetic autonomic neuropathy is a complication of poor glycemic control [10,11]. It is therefore thought that since erythropoietin production and release is regulated in part by autonomic nervous system, the results suggest that erythropoietin production could be prematurely impaired in patients with poor glycemic control. Other factors which have been reported to increase the risk of anemia include; systemic inflammation [12]; damage to renal achitecture produced by chronic hyperglycemia and consequent formation of advanced glyction end products $[1,2,13]$; and depressed androgen levels induced by diabetes [14]. It is speculated that these conditions may be aggravated in poorly controlled diabetes than in controlled diabetes. Our finding therefore suggest that a reduction of blood glucose levels and the targeting of acceptable glycated haemoglobin levels would help reduce the risk of anemia in the diabetic population.

Our data also indicated greater odds of anemia in patients of age $\geq 60$ yrs when compared to those of age $<$ $60 \mathrm{yrs}$. This result was expected since age is associated with both hemoglobin levels and anemia irrespective of health status $[15,16]$. Increased odds of anemia has also been found in previous studies $[5,17,18]$ among older diabetic patients and consistent with the present findings. These findings indicate the need for proper diabetic care and management for diabetic senior citizens, who have limited food choices and are more vulnerable to irondeficiency anemia. Furthermore, our data indicated no significant difference in the risk of anemia between males and females. This finding is consistent with a previous study [18]. However, in another study involving patients with moderate levels of renal defficiencies, gender difference in the risk of anemia has been reported [5].

In conclusion, a high incidence of anemia was observed in diabetics without renal insufficiency. Our data also suggested that poor glycemic control and old age are associated with the incidence of anemia in diabetic patients with normal renal function. Correction of anemia may have a significant role in prevention of other diabetic complications, thus we recommend that treatment criteria for diabetes should include routine hematological tests and take into consideration the inevitable consequences of aging, and poor glycemic control, in order to make optimal therapeutic decisions for the treatment of diabetes mellitus in adults.

\section{REFERENCES}

[1] Thomas, M.C., MacIsaac, R.J., Tsalamandris, C., Molyneaux, L., Goubina, I., Fulcher, G., Yue, D. and Jerums, G. (2004) The burden of anaemia in type 2 diabetes and the role of nephropathy: A cross-sectional audit. Nephrology Dialysis Transplantation, 19, 1792-1797.

doi:10.1093/ndt/gfh248

[2] Bosman, D.R., Winkler, A.S., Marsden, J.T., Macdougall, I.C. and Watkins, P.J. (2001) Anaemia with erythropoietin deficiency occurs early in diabetic nephropathy. Diabetes Care, 24, 495-499. doi:10.2337/diacare.24.3.495

[3] Dikow, R., Schwenger, V., Schomig, M. and Ritz, E. (2002) How should we manage anaemia in patients with diabetes? Nephrology Dialysis Transplantation, 17, 67-72. doi:10.1093/ndt/17.suppl_1.67

[4] Ishimura, E., Nishizawa, Y., Okuno, S., Matsumoto, N., Emoto, M., Inaba, M., Kawagishi, T., Kim, C.W. and Morii, H. (1998) Diabetes mellitus increases the severity of anaemia in non-dialysed patients with renal failure. Journal of Nephrology, 11, 83-86.

[5] El-achkar, T.M., Ohmit, S.E., Mccullough, P.A., Crook, E.D., Brown, W.W., Grimm, R., Bakris, G.I., Keane, W.F. and Flack, J.M. (2005) Higher prevalence of anemia with diabetes mellitus in moderate kidney insufficiency: The Kidney Early Evaluation Program. Kidney International, 67, 1483-1488. doi:10.1111/j.1523-1755.2005.00226.x

[6] Craig, K.J., Williams, J.D., Riley, S.G., Smith, H., Owens, D.R., Worthing, D., Cavill, I. and Phillips, A.O. (2005) Anemia and diabetes in the absence of nephropathy. Diabetes Care, 28, 1118-1123. doi:10.2337/diacare.28.5.1118

[7] Kojima, K. and Totsuka, Y. (1995) Anemia due to reduced serum erythropoietin concentration in non-uremic diabetic patients. Diabetes Research and Clinical Practice, 27, 229-233. doi:10.1016/0168-8227(95)01042-C

[8] World Health Organization (1968) Nutritional anaemia Report of a WHO Scientific Group, WHO, Geneva, 1968.

[9] Bonakdaran, S., Gharebaghi, M. and Vahedian, M. (2011) Prevalence of anemia in type-2 diabetes and role of renal involvement. Saudi Journal of Kidney Diseases and Transplantation, 22, 286-290.

[10] Ahmed, A.M., Hussein, A. and Ahmed, N.H. (2000) Diabetic autonomic neuropathy. Saudi Medical Journal, 21, 1034-1037.

[11] Toyry, J.P., Niskanen, L.K., Mantyseari, M.J., Lansimies, E.A. and Uusitupa, M.I. (1996) Occurrence, predictors and clinical significance of autonomic neuropathy in NIDDM. Ten year follow-up from the diagnosis. Diabetes, 45, 308-315. doi:10.2337/diabetes.45.3.308

[12] Cottone, S., Lorito, M.C., Riccobene, R., Nardi, E., Mule, G., Buscemi, S., Geraci, C., Guarneri, M., Arsena, R. and Cerasola, G. (2008) Oxidative stress, inflammation and cardiovascular disease in chronic renal failure. Journal of 
Nephrology, 21, 175-179

[13] Schuster, S.J., Koury, S.T., Bohrer, M., et al. (1992) Cellular sites of extrarenal and renal erythropoietin production in anaemic rats. British Journal of Haematology, 81, 153-159. doi:10.1111/j.1365-2141.1992.tb08200.x

[14] Andersson, B., Marin, P., Lissner, L., et al. (1994) Testosterone concentrations in women and men with NIDDM. Diabetes Care, 17, 405-411. doi:10.2337/diacare.17.5.405

[15] Salive, M.E., Cornoni-Huntley, J., Guralnik, J.M., Phillips, C.L., Wallace, R.B., Ostfeld, A.M. and Cohen, H.J. (1992) Anemia and hemoglobin levels in older persons: Relationship with age, gender, and health status. Journal of the American Geriatrics Society, 40, 489-496.

[16] Gaskell, H., Derry, S., Moore, R.A. and McQuay, H.J. (2008) Prevalence of anaemia in older persons: Systematic review. BMC Geriatrics, $8,1$. doi:10.1186/1471-2318-8-1

[17] Goldhaber, A., Ness-Abramof, R. and Ellis, M.H. (2009) Prevalence of anemia among unselected adults with diabetes mellitus and normal serum creatinine levels. Endocrine Practice, 15, 714-719. doi:10.4158/EP09119.ORR

[18] Ahmed, A.T., Go, A.S., Warton, E.M., Parker, M.M. and Karter, A.J. (2010) Ethnic differences in anemia among patients with diabetes mellitus: The Diabetes Study of Northern California (DISTANCE). American Journal of Hematology, 85, 57-61. 